\title{
An Object-based Modified Conditional Random Field for Landslide Mapping Using GaoFen-1 Image
}

\author{
Fangfang Shao, Xiuguo Liu, Qihao Chen", Linlin Li and Yonglin Shen \\ Faculty of information engineering, China University of Geosciences, Wuhan, \\ P.R. China \\ chenqihao@cug.edu.cn
}

\begin{abstract}
Landslide mapping is an important part of landslide research for natural hazard analysis. A lot of studies have been made for object-based image analysis of landslide mapping, with increasing resolution of satellite image in recent years. However, the propagation of mis-classification from super or adjacent objects limits the improvement of landslide mapping accuracy. In this paper, we present a new object-based conditional random field $(C R F)$ combining the context information to perform landslide mapping accurately. The CRF model is built based on the image objects (or segments) graph from object-based classification. And its pairwise potential is constructed by substituting the posterior probability from support vector machine (SVM) classification for the feature vectors. We investigate the potential benefits of using segment graph structure and the modifying potential of the CRF model through experiments conducted on GaoFen-1 (GF1) satellite image. The results reveal the proposed method has efficient performance of improving landslide mapping accuracy.
\end{abstract}

Keywords: conditional random field (CRF); landslide mapping; object-based classification; support vector machine (SVM); GaoFen-1 (GF-1)

\section{Introduction}

Landslides are a major hazard in almost all mountainous regions of the world. Landslide mapping is a critical requirement in pre- and post- disaster hazard analysis [12]. Recently, Object-based image analysis has been used for landslide mapping by some studies [3-5] through classification and change detection with high resolution remote sensing images and aerial photographs [6-7]. Object-based image analysis represents a more advantageous approach for analysing high-resolution data because image pixels can be meaningfully grouped into networked homogeneous objects [5]. Another benefit is that object-based methods can make use of any kind features deducible from an image segment such as shape, texture, and context information. And then, numerous studies describe context information by using image-objects hierarchy [8-9] and adjacency [4] in the classification process [10]. Up to now, some landslide mapping methods were also combined to context information in this limited way [4-5], which suffers from the propagation of mis-classification information from super or adjacent objects.

A general framework of modelling context information is using Markov random field (MRF) approaches [11-12], which is a classical probabilistic graphical model. However, the interaction between neighbouring image sites (pixels or objects) is restricted to class labels, whereas the features extracted from different image sites are assumed to be conditionally independent [13]. Conditional Random Fields (CRFs) [14] provides a more general framework that can model interactions between the labels and the arbitrary features from different image sites. CRFs have become a standard technique for

* Corresponding Author 
considering context information in classification, in particular for image classification [15].

Applications of the CRF framework differ in definition of the graph structure and in the specification of potentials [15]. For the graph structure, CRFs were built on pixels [13, 16-18] or image objects (segments or superpixels) [19-21]. Compared to pixel-based classification, using image-objects as basic units allows to reduce significantly the graph size and to accelerate inference. For the potentials, Potts model is generally used to construct the pairwise potentials, which is one of the important type of potentials. To avoid too many training parameters in the model, the contrast-sensitive Potts model based on Euclidean distance of the feature vectors has been used and modified [13, 18, 22]. In practice, the application scenes of landslide mapping are more complicated than the general classification. Thus, an appropriate pairwise potential is needed to improve the accuracy of landslide mapping.

In this paper, we introduce the CRF into landslide mapping, and propose an objectbased modified CRF to perform landslide mapping using remote sensing image. This CRF model combines context information based on object-based classification. We construct the pairwise potentials by substituting posterior probability from SVM classification for the feature vectors to improve accuracy of landslide mapping.

\section{Method Description Overview of Our Model}

\subsection{Overview of Our Model}

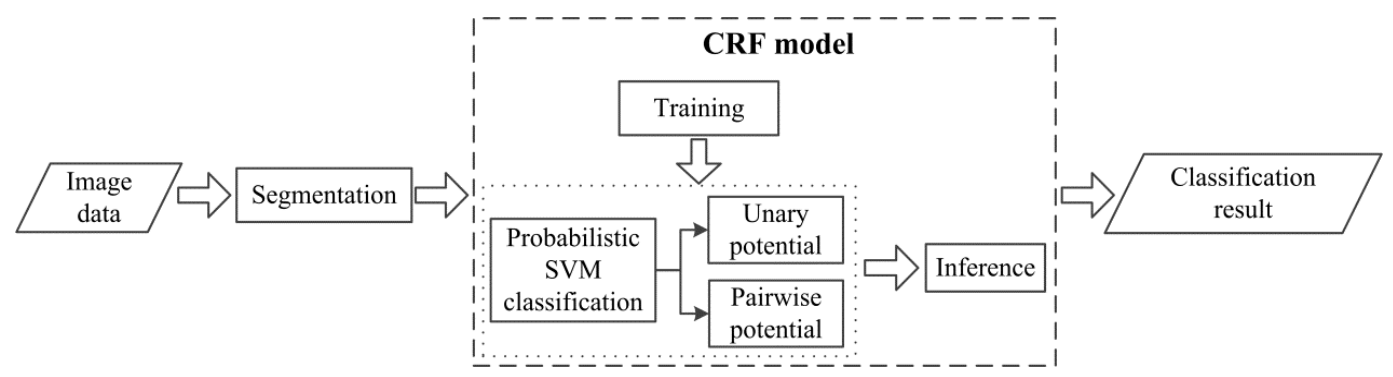

Figure 1. Flowchart of the Proposed Approach

The flowchart of the proposed object-based CRF classification method is shown in Figure 1. As shown in Figure 1, the CRF model works on irregular graph built on segments after segmentation. CRF is defined as follows. Let $G=(V, E)$ be the graph structure where $V$ is the node set for given image data $\boldsymbol{x}$ and $\mathrm{E}$ is the edge set between adjacent nodes. In our case, each node $i \in V$ correspond to a segment of the image and each edge $(i, j) \in E$ is a link between two adjacent nodes $i$ and $j$. Let $\boldsymbol{y}=\left\{y_{i}\right\}$ be the class labels set for all nodes, and has the same number of elements as $V$. CRFs are discriminative model that directly describe the posterior probability of the label sets y given the entire observation data $\boldsymbol{x}$ [12]. Then, the posterior probability distribution $P(\boldsymbol{y} \mid \boldsymbol{x})$ of CRF can be modelled as:

$$
P(\boldsymbol{y} \mid \boldsymbol{x})=\frac{1}{Z(\boldsymbol{x})} \exp \left[\sum_{i \in V} A_{i}\left(y_{i}, \boldsymbol{x}\right)+\alpha \sum_{i \in V} \sum_{j \in L_{i}} I S\left(y_{i}, y_{j}, \boldsymbol{x}\right)\right]
$$




$$
Z(\boldsymbol{x})=\sum_{y_{i} \in \boldsymbol{y}} \exp \left[\sum_{i \in V} A_{i}\left(y_{i}, \boldsymbol{x}\right)+\alpha \sum_{i \in V} \sum_{j \in L_{i}} I S\left(y_{i}, y_{j}, \boldsymbol{x}\right)\right]
$$

In Equation (1), $L_{i}$ is the neighbourhood of node $i . A_{i}\left(y_{i}, \boldsymbol{x}\right)$ is the unary potential, which builds a relationship between the label $y_{j}$ and the data $\left.\boldsymbol{x} . I_{i}, y_{j}, \boldsymbol{x}\right)$ is the pairwise potential, which builds dependencies between the label $y_{i}$ and $y_{j}$ of single node $i$ and its neighbourhoods $j$ and the data $\boldsymbol{x} . Z(\boldsymbol{x})$ is a normalized constant called the partition function.

\subsection{Definition of the Graph Structure}

In remote sensing image classification, CRFs is usually built on the simple regular graph based on pixels(Figure 2(a)).However, in fact, segments (superpixels or objects), created from good segmentation results, are semantically more meaningful than pixels so that they are more helpful for accurate classification results [21]. For landslide, segment representation can decreases the variance of spectral values between landslide objects by averaging the pixels within the object [23]. We therefore exploited an irregular graph on segments to implement CRF (see Figure 2(b)).

In our case, to get the segment graph and construct the object-based CRF, an oversegmentation was performed into objects through a segmentation algorithm named Fractal Net Evolution Approach (FNEA) using eCognition software[22] (see Figure 1). FNEA involves generating a hierarchical segmentation at various scales that produce satisfying results with respect to the desired geometrical accuracy of image-object boundaries, which starts with a single pixel and a pairwise comparison of its neighbours with the aim of minimizing the resulting summed heterogeneity [24].

An object or segment is considered to be an undivided unit in which all pixels have the same pattern or label. Each node corresponds to an object or segment, and the edges indicate an adjacency relationship among objects.

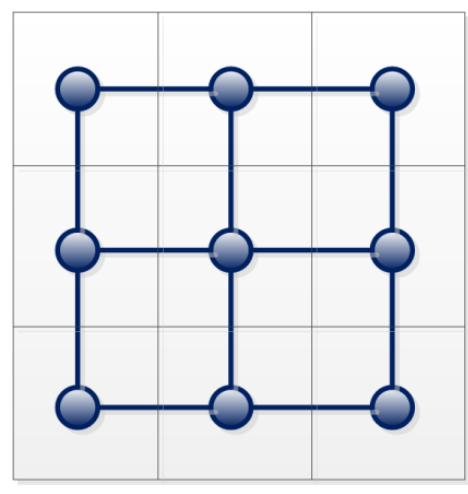

(a)

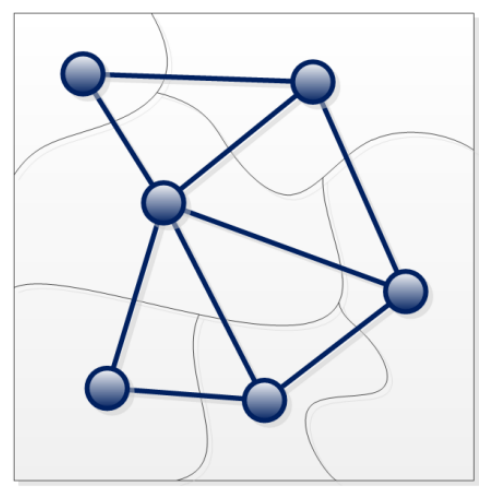

(b)

Figure 2. (a) Regular Graph Based on Pixels, (b) Irregular Graph Based on Segments 


\subsection{Unary Potential}

The unary potential $A_{i}\left(y_{i}, \boldsymbol{x}\right)$ in Equation (1) is frequently modelled by using the posterior probability of label ${ }^{y_{i}}$ given the data $x$. It can be represented as:

$$
A_{i}\left(y_{i}, \boldsymbol{x}\right)=\log \left[p\left(y_{i} \mid \boldsymbol{x}\right)\right]
$$

The posterior probability $p\left(y_{i} \mid \boldsymbol{x}\right)$ can be calculated by using a classifier. The most widely used classifier is Maximum Likelihood Classifier (MLC), which restrict the data to be assumed to have Gaussian distribution. SVM has a free assumption and ability to find complex classification boundaries with good generalization performance [11]. SVM classifier also has been used to model the unary potential of CRF and exhibited excellent performance [17].In this paper, we adopt SVM classifier to calculate $p\left(y_{i} \mid \boldsymbol{x}\right)$ and in this manner SVM is integrated into the unary potential.

\subsection{Pairwise Potential}

Pairwise potential incorporates the contextual information explicitly by modelling the interaction between nodes $i$ and adjacent nodes $j$ through considering the label and the observed data $\boldsymbol{x}$. Conventional CRFs always incorporate some common features, such as spectral and texture features, to model the pairwise potential. In the proposed ModifiedCRF model, posterior probability derived from SVM classifier is used to model the pairwise potential, instead of using those features directly.

The posterior probability is incorporated into a modified version of contrast-sensitive Potts model [18] in this paper. The pairwise potential can then be written as:

$$
I S\left(y_{i}, y_{j}, \boldsymbol{x}\right)=\left\{\begin{array}{cl}
\alpha & \text { if } y_{i}=y_{j} \\
\alpha \cdot\left\{1-\exp \left[-\left\|\Delta \boldsymbol{p}_{i j}(\boldsymbol{y} \mid \boldsymbol{x})\right\|^{2}\right]\right\} & \text { if } y_{i} \neq y_{j}
\end{array}\right.
$$

In Equation (4), $\alpha$ is a weighting parameter for controlling the contribution of the

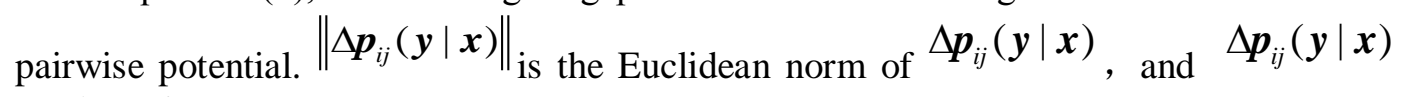
can be written as:

$$
\Delta \boldsymbol{p}_{i j}(\boldsymbol{y} \mid \boldsymbol{x})=\left\{\begin{array}{c}
\left|p_{i}\left(y_{1} \mid \boldsymbol{x}\right)-p_{j}\left(y_{1} \mid \boldsymbol{x}\right)\right| \\
\left|p_{i}\left(y_{2} \mid \boldsymbol{x}\right)-p_{j}\left(y_{2} \mid \boldsymbol{x}\right)\right| \\
\ldots \\
|| p_{i}\left(y_{K} \mid \boldsymbol{x}\right)-p_{j}\left(y_{K} \mid \boldsymbol{x}\right) \mid
\end{array}\right.
$$

In Equation (5), $\mathrm{K}$ is the number of classes. $\left\langle\boldsymbol{p}_{i j}(\boldsymbol{y} \mid \boldsymbol{x})\right.$ represents the component-wise differences of the posterior probability belonging to each class derived from SVM classifier between node $i$ and its adjacent node $j$. Each component of $\Delta \boldsymbol{p}_{i j}(\boldsymbol{y} \mid \boldsymbol{x})$ is in the same scale because the posterior probability can normalize the variability of different features. $\left\|\Delta \boldsymbol{p}_{i j}(\boldsymbol{y} \mid \boldsymbol{x})\right\|_{\text {represents the quantified distance magnitude between the adjacent }}^{2}$ nodes. The greater $\left\|\Delta \boldsymbol{p}_{i j}(\boldsymbol{y} \mid \boldsymbol{x})\right\|^{2}$ is, higher the difference of adjacent nodes.

In this pairwise potential, if the node $i$ and its adjacent node $j$ have similar posterior probability, then the value of $\exp \left[-\left\|\Delta \boldsymbol{p}_{i j}(\boldsymbol{y} \mid \boldsymbol{x})\right\|^{2}\right]$ is close to 1 , that is the probability of 
them belonging to the same label is great. In contrast, if node $i$ and its adjacent node $j$ have different posterior probability, then the probability of them belonging to same label is small.

\subsection{Training and Inference}

Weighting parameter $\alpha$ involved in CRF is crucial to determine the importance of pairwise potential, and is estimated by cross validation on the training data [25]. Since Loopy Belief Propagation (LBP) [26] is a standard technique with good performance [27] for performing probability propagation in graphs containing cycles, it is used for inferring probabilities of the nodes belonging to the different classes.

\section{Results and Discussion}

\subsection{GF-1 Data and Experiment Results}

A case study is carried out by using a subset of GaoFen-1 (GF-1) satellite image (643×643 pixels) acquired in 2013 from Wenchuan county of Sichuan province in China. The GF-1 satellite images consist of $2 \mathrm{~m}$ spatial resolution panchromatic image and $8 \mathrm{~m}$ spatial resolution multispectral image. To take advantage of both panchromatic and multispectral images, pan-sharpen fusion method is performed to create high spatial resolution multispectral image. Figure 3 displays the experimental satellite fusion image and the reference landslides inventory map. Two classes: landslide and non-landslide were labeled in our case study. Training samples have been randomly selected for each class from the ground-truth data.

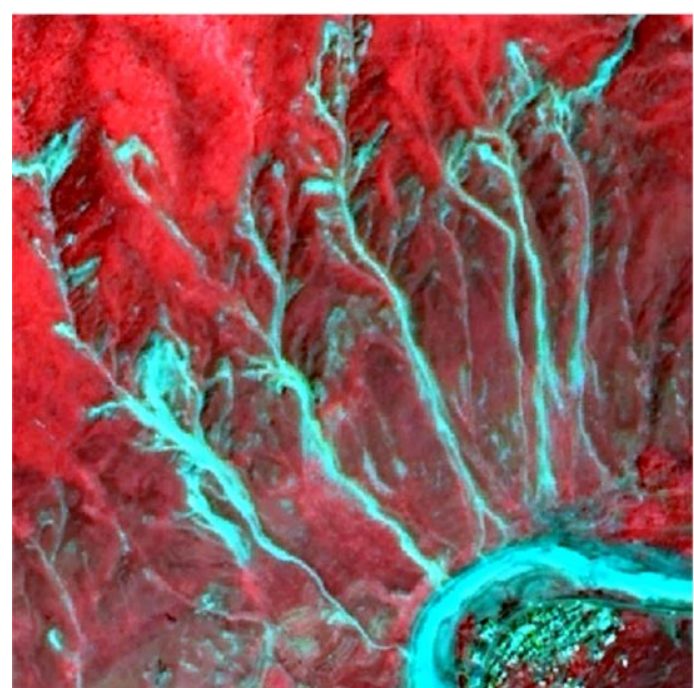

(a)

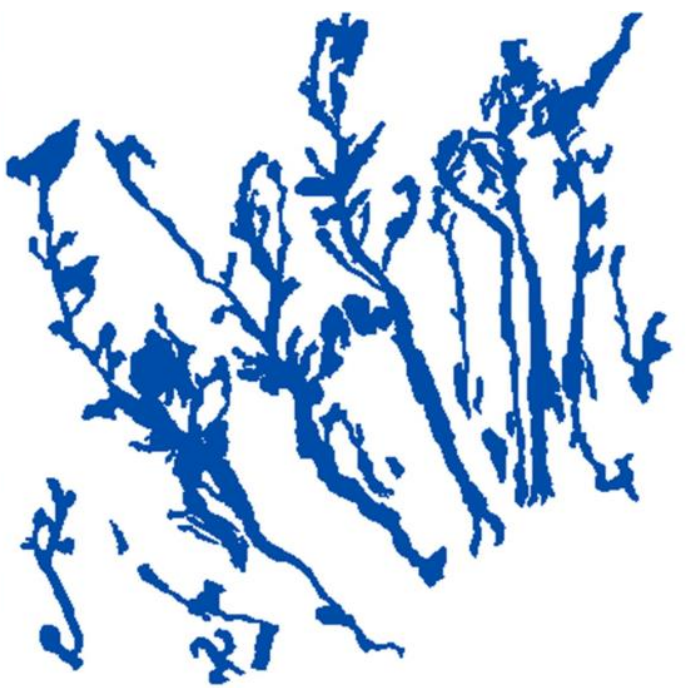

(b)

Figure 3. (a) Original RGB image, (b) Ground-truth Image

To evaluate the performance of proposed object-based CRF, we investigate the influence of (1) using irregular graph structure on image segments, (2) applying modified CRF to the segment graph structure. Therefore, we compared several classifiers with two different formulations: on image pixels level and on image objects level. Those classifiers are (1) non-contextual SVM, (2) the CRF model proposed by Zhang and Jia [18], named simplified-CRF, which uses image general features to model the pairwise potential, and (3) our modified-CRF.

In the proposed method, over-segmentations by FNEA were implemented with small scale to create objects for recognizing smaller landslides. The values of segmentation 
parameters were determined by experiment. The scale is 10 , the spectral weight is 0.9 , and the compactness is 0.5. The segmentation result is shown in Figure 4.

Then, spectral features, slope feature, and texture features were selected as input features for SVM classification. Spectral features are four spectral bands of the image (NIR, red, green and blue). The texture features include homogeneity, contrast, entropy, correlation, mean, and standard deviation, and they were computed from the grey level co-occurrence matrix. The mean and standard deviation of four spectral bands and all above-mentioned texture features were extracted from image segments and were applied to the object-based classification experiments.

The non-contextual SVM classifier is used for building the unary potential and pairwise potential of the proposed modified-CRF. This classifier applies a radial basis function (RBF) as the kernel, and the appropriate values of the parameters $C$ and $\gamma$ were determined by the cross validation.

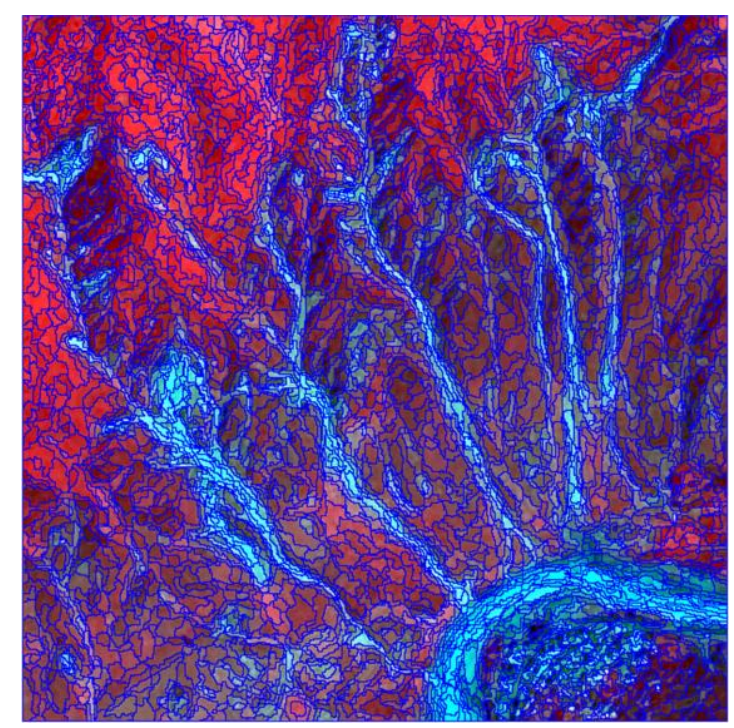

Figure 4. Segmentation Result Image

After modelling the unary potential and pairwise potential of CRF, weighting parameter $\alpha$ is trained by cross validation, and then LBP algorithm is performed to inference the classification results. 


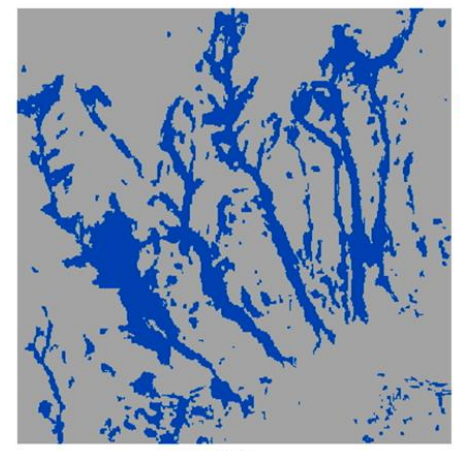

(a)

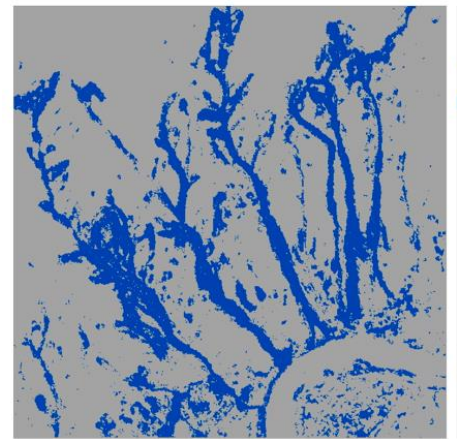

(d)

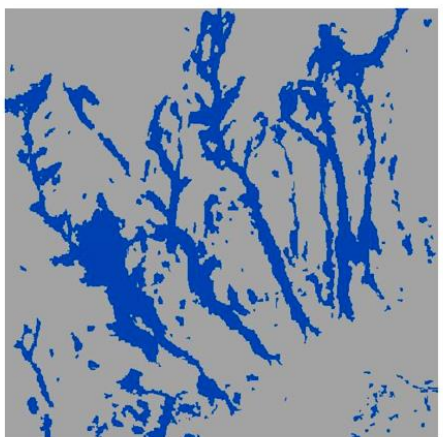

(b)

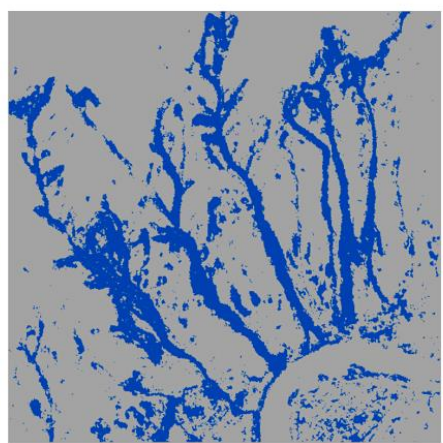

(e)

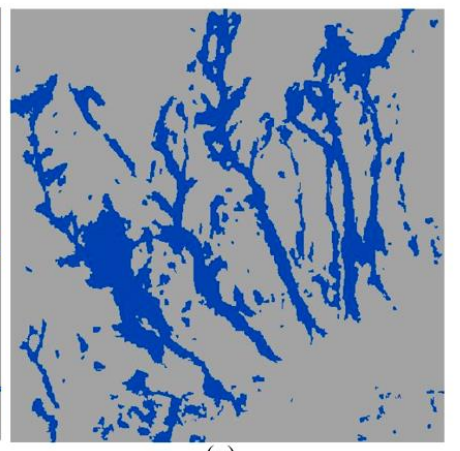

(c)

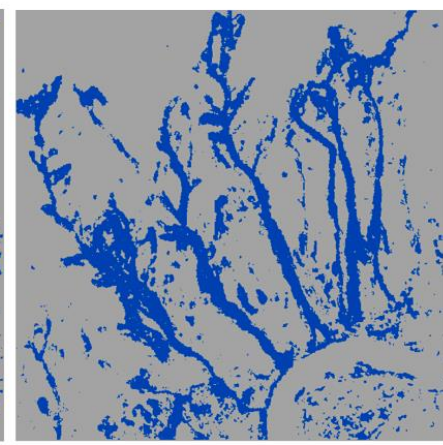

(f)

Figure 5. Experimental Results: (a) Object-based SVM, (b) Object-based Simplified-CRF Model, (c) Object-based Modified-CRF Model, (d) Pixelbased SVM, (e) Pixel-based Simplified-CRF Model, (f) Pixel-based ModifiedCRF Model

The classification results by different methods are shown in Figure 5. It can be seen that the proposed object-based modified-CRF model acquired the best classification results (Figure 5c). Both our modified-CRF and object-based simplified-CRF achieved smoother results than SVM. The combination of object-based classification and graphical model, i.e., adapting the graphical model to the segment graph structure, make satisfactory result compared with other methods. Furthermore, for pixel-based classification, residential areas at lower right of the image are almost mis-classified as landslides, while only small residential areas are mis-classified as landslides in objectbased classifications. For pixel-based classification, simplified-CRF and our modifiedCRF did not achieve distinct continuous result compared to pixel-based SVM. By comparison, the proposed method obtained smoother result and filtered out more false positives.

\subsection{Accuracy Assessment}

In order to carry out a quantitative evaluation for the performance of different classifiers, confusion matrices were generated for all the classification results. Overall accuracy (OA), Kappa coefficient and three accuracy indices of the mapped landslides (the producer's accuracy, user's accuracy, and their average accuracy) are derived from each error matrix and are presented in Table 1. As can be seen from Table 1, the best OA and Kappa coefficient is obtained by our object-based modified-CRF with $92.38 \%$ and 0.7751. For both object-based methods and pixel-based methods, SVM algorithm had the lowest OA and Kappa. Modified-CRF gets slightly higher OA and higher Kappa than simplified-CRF on image object level as well as on image pixels level. When considering the influence of graph structure, it is worth noting that each graphical model on object level, yield consistently higher OA and Kappa, in comparison to the corresponding model 
on pixels level. The graphical model on image segments improved the overall accuracy about $2 \%$ and the kappa by about 0.05 .

In terms of the accuracy for landslide, all the object-based methods significantly improve landslide accuracy than pixel-based methods by increasing producer accuracy, user accuracy and mean accuracy about $4 \% \sim 6 \%$. For both object-based classification and pixel-based classification, both modified-CRF and simplified-CRF gets slightly lower producer accuracy than SVM, but gets higher user accuracy and mean accuracy. The proposed modified-CRF has higher producer accuracy, user accuracy and mean accuracy than Simplified-CRF.

Table 1. Classification Accuracy Comparison of the Proposed Method with Other Methods

\begin{tabular}{|c|c|c|c|c|c|c|}
\hline \multirow{2}{*}{\multicolumn{2}{|c|}{ Methods }} & \multicolumn{3}{|c|}{ Landslide accuracy (\%) } & \multirow{2}{*}{$\begin{array}{c}\text { Overall } \\
\text { accuracy } \\
(\%)\end{array}$} & \multirow[b]{2}{*}{ Kappa } \\
\hline & & $\begin{array}{l}\text { Producer } \\
\text { accuracy }\end{array}$ & $\begin{array}{c}\text { User } \\
\text { accuracy }\end{array}$ & $\begin{array}{c}\text { Mean } \\
\text { accuracy }\end{array}$ & & \\
\hline \multirow{3}{*}{$\begin{array}{l}\text { Object-based } \\
\text { Classification }\end{array}$} & SVM & 83.23 & 79.61 & 81.42 & 91.75 & 0.7616 \\
\hline & $\begin{array}{l}\text { Simplified- } \\
\text { CRF }\end{array}$ & 82.50 & 81.27 & 81.885 & 92.19 & 0.7690 \\
\hline & $\begin{array}{c}\text { Modified- } \\
\text { CRF }\end{array}$ & 83.13 & 81.61 & 82.37 & 92.38 & 0.7751 \\
\hline \multirow{3}{*}{$\begin{array}{l}\text { Pixel-based } \\
\text { Classification }\end{array}$} & SVM & 77.67 & 75.93 & 76.80 & 89.92 & 0.7036 \\
\hline & $\begin{array}{l}\text { Simplified- } \\
\text { CRF }\end{array}$ & 77.56 & 76.42 & 76.99 & 90.04 & 0.7063 \\
\hline & $\begin{array}{l}\text { Modified- } \\
\text { CRF }\end{array}$ & 77.62 & 77.24 & 77.43 & 90.28 & 0.7124 \\
\hline
\end{tabular}

From the aforementioned analysis, for landslide mapping, the object-based models can help to improve producer accuracy and user accuracy, compared to the pixel-based models. The modified CRF model can help to improve the user accuracy and keep enhancing producer accuracy compared to Simplified-CRF.

\section{Conclusion}

In this paper, we have proposed an object-based modified CRF model to perform landslide mapping accurately. This CRF model was built based on image segments, which allows incorporating spatial context information among image neighbouring objects. The pairwise potential of the CRF has been constructed with the posterior probability instead of image objects features from the contrast-sensitive Potts model, to improve the accuracy and stability. The results show that the proposed CRF model has better performance on landslide detection and removal of false positives in comparison to models built on image pixels. The modification of pairwise potential leads to a further increase in accuracy. It is reasonable to improve landslide mapping accuracy by using the segment graph structure to construct CRF model and integrating the posterior probability derived from SVM classifier into pairwise potential of CRF.

The classification results heavily depend on the segmentation and small landslide objects with sub-segment size cannot be detected. The image segmentation using scale optimization is a major issue we should consider in our future work.

\section{Acknowledgments}

This work is supported in part by the National Natural Science Foundation of China under Grant No. 41301477, 41471355. The authors would like to acknowledge K. 
Murphy \& M. Schmidt for providing CRF Toolbox called "UGM: Matlab code for undirected graphical models": www.cs.ubc.ca/ murphyk/Software/CRF/crf.html.

\section{References}

[1] M. Ao, J. Zhu, C. Wang and X. Yu, "Identification of Landslide Region Based on Topographic and Change Information", International Journal of Signal Processing, Image Processing and Pattern Recognition, vol. 7, no. 1, (2014), pp. 121-134.

[2] S. K. Bejo and M. Petrou, "Elastic image registration for landslides monitoring", International Journal of Signal Processing, Image Processing and Pattern Recognition, vol. 3, no. 3, (2010), pp. 71-86.

[3] A. Stumpf and N. Kerle, "Object-oriented mapping of landslides using Random Forests", Remote Sensing of Environment, vol. 115, no. 10, (2011), pp. 2564-2577.

[4] G. Cheng, L. Guo, T. Zhao, J. Han, H. Li and J. Fang, "Automatic landslide detection from remotesensing imagery using a scene classification method based on BoVW and pLSA", International Journal of Remote Sensing, vol. 34, no. 34, (2013), pp. 45-59.

[5] S. Lee, K. Y. Song, H. J. Oh and J. Choi, "Detection of landslides using web-based aerial photographs and landslide susceptibility mapping using geospatial analysis", International Journal of Remote Sensing, vol. 33, no. 16, (2012), pp. 4937-4966.

[6] L. Durieux, E. Lagabrielle and A. Nelson, "A method for monitoring building construction in urban sprawl areas using object-based analysis of Spot 5 images and existing GIS data", Isprs Journal of Photogrammetry \& Remote Sensing, vol. 63, no. 4, (2008), pp. 399-408.

[7] N. Campos, R. Lawrence, B. Mcglynn and K. Gardner, "Effects of LiDAR-Quickbird fusion on objectoriented classification of mountain resort development", Journal of Applied Remote Sensing, vol. 4, no. 11, (2010), pp. 2816-2832.

[8] J. P. O’Neil-Dunne, S. Macfaden and K. C. Pelletier, "Incorporating contextual information into objectbased image analysis workflows", ASPRS 2011 Annual Conference, Milwaukee, America, (2011).

[9] D. Liu, M. Kelly and P. Gong, "A spatial-temporal approach to monitoring forest disease spread using multi-temporal high spatial resolution imagery", Remote Sensing of Environment, vol. 101, no. 2, (2006), pp. 167-180.

[10] X. Jia and J. A. Richards, "Managing the Spectral-Spatial Mix in Context Classification Using Markov Random Fields", IEEE Geoscience \& Remote Sensing Letters, vol. 5, no. 2, (2008), pp. 311-314.

[11] T. Hoberg, F. Rottensteiner and C. Heipke, "Classification of multitemporal remote sensing data using conditional random fields", In Proceedings of the 6th IAPR Workshop on Pattern Recognition in Remote Sensing (PRRS), Istanbul, Turkey, (2010).

[12] S. Kumar and M. Hebert, "Discriminative Random Fields", International Journal of Computer Vision, vol. 68, no. 2, (2006), pp. 179-201.

[13] J. Niemeyer, F. Rottensteiner and U. Soergel, "Contextual classification of lidar data and building object detection in urban areas”, Isprs Journal of Photogrammetry \& Remote Sensing, vol. 87, no. 1, (2014), pp. $152-165$.

[14] T. Hoberg, F. Rottensteiner, R. Queiroz Feitosa and C. Heipke, "Conditional Random Fields for Multitemporal and Multiscale Classification of Optical Satellite Imagery", Geoscience \& Remote Sensing IEEE Transactions On, vol. 53, no. 2, (2015), pp. 659-673.

[15] Y. Zhong, X. Lin and L. Zhang, "A support vector conditional random fields classifier with a Mahalanobis distance boundary constraint for high spatial resolution remote sensing imagery", Selected Topics in Applied Earth Observations and Remote Sensing, IEEE Journal of, vol. 7, no. 4, (2014), pp. 1314-1330.

[16] G. Zhang and X. Jia, "Simplified Conditional Random Fields With Class Boundary Constraint for Spectral-Spatial Based Remote Sensing Image Classification”, IEEE Geoscience \& Remote Sensing Letters, vol. 9, no. 5, (2012), pp. 856-860.

[17] X. Su, C. He, Q. Feng, X. Deng and H. Sun, “A Supervised Classification Method Based on Conditional Random Fields With Multiscale Region Connection Calculus Model for SAR Image", Geoscience \& Remote Sensing Letters IEEE, vol. 8, no. 3, (2011), pp. 497-501.

[18] J. Wegne, U. Soergel and B. Rosenhahn, "Segment-based building detection with conditional random fields", Urban Remote Sensing Event, Munich, Germany, (2011).

[19] O. Besbes, N. Boujemaa and Z. Belhadj, "Contextual Classification of High-Resolution Satellite Images", Computational Intelligence for Image Processing, Nashville, TN, (2009).

[20] J. Shotton, J. Winn, C. Rother and A. Criminisi, "TextonBoost for Image Understanding: Multi-Class Object Recognition and Segmentation by Jointly Modeling Texture, Layout, and Context", International Journal of Computer Vision, vol. 81, no. 1, (2009), pp. 2-23.

[21] T. Lahousse, K. T. Chang and Y. H. Lin, "Landslide mapping with multi-scale object-based image analysis - a case study in the Baichi watershed, Taiwan", Natural Hazards \& Earth System Sciences, vol. 11, no. 10, (2011), pp. 2715-2726.

[22] T. Blaschke, "Object based image analysis for remote sensing", Isprs Journal of Photogrammetry \& Remote Sensing, vol. 65, no. 1, (2010), pp. 2-16. 
[23] T. Lahousse, K. T. Chang and Y. H. Lin, "Landslide mapping with multi-scale object-based image analysis - a case study in the Baichi watershed, Taiwan", Natural Hazards \& Earth System Sciences, vol. 11, no. 10, (2011), pp. 2715-2726.

[24] G. J. Hay, T. Blaschke, D. J. Marceau and A. Bouchard, "A comparison of three image-object methods for the multiscale analysis of landscape structure", ISPRS Journal of Photogrammetry and Remote Sensing, vol. 57, no. 5, (2003), pp. 327-345.

[25] M. Y. Yang and W. Förstner, "A hierarchical conditional random field model for labeling and classifying images of man-made scenes", IEEE International Conference on Computer Vision Workshops, ICCV 2011 Workshops, Barcelona, Spain, (2011).

[26] J. Pearl, "Probabilistic reasoning in intelligent systems: Networks of plausible reasoning", Morgan Kaufmann Publishers, Los Altos, (1988).

[27] S. V. N. Vishwanathan, N. N. Schraudolph, M. W. Schmidt and K. P. Murphy, "Accelerated training of conditional random fields with stochastic gradient methods", International Conference on Machine Learning. ACM, (2006), pp. 969-976

\section{Authors}

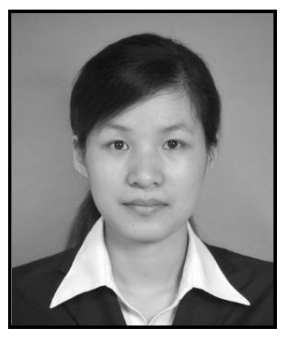

Fangfang Shao, is a Master student in Faculty of Information Engineering, China University of Geosciences, Wuhan, China. Her research interests include object-based change detection and monitoring geological disasters utilized remote sensing technology. 